\title{
Decision making in an uncertain environment: An application of ROC analysis for credit scoring in the mobile telephone market
}

Received (in revised form): 15th January, 2004

\section{Marc Hoogenberg}

works as a business consultant at Paul Postma Marketing Consultancy in the Netherlands. He provides solutions and training in data analysis and data modelling problems for customer relationship management (CRM).

\section{Sander auf dem Brinke}

works as a business consultant at Paul Postma Marketing Consultancy in the Netherlands. He advises in analytical CRM projects and has several years of experience in a variety of sectors, including telecommunications.

Abstract This paper describes the use of relative operating characteristics (ROC) analysis for decision making under uncertain circumstances. It is presented in the framework of credit scoring for a mobile phone operator.

Sander auf dem Brinke Paul Postma Marketing Consultancy B.V.,

Edisonbaan 14A, $3439 \mathrm{MN}$, Nieuwegein,

The Netherlands.

Tel: +31 (0)30 2598460; Fax: $+31(0) 302598484$; e-mail:

sander.aufdembrinke@ ppmc.nl

\section{INTRODUCTION}

Now that the cards have been dealt, and the market share of mobile telephone operators has converged to a steady state, their marketing focus is shifting from the brute force acquisition of new customers to a more refined strategy. It is becoming more and more important to focus on customers (potentially) generating high value. A problem that perpetuates, however, is that of 'noncredible' customers. Every year, customers who can, or will, no longer pay their monthly subscription fee and/or telephone bill are a large source of costs for mobile telephone operators. Within the data mining community, the problem of predicting and detecting (deliberate) refusal of payments has become a major topic in applied research. It is often called the credit-scoring problem. This paper discusses the use of relative operating characteristics (ROC) analysis in the setting of credit scoring for a mobile phone operator. It shows that ROC analysis is a particularly suitable instrument for decision making in uncertain situations like the mobile telephone market.

\section{PROBLEMS IN CREDIT SCORING}

Credit scoring can be applied at two stages. ${ }^{1}$ The behaviour of existing customers can be analysed to detect credit risk. On the other hand, at the application stage, new potential customers (prospects) can be assessed in order to predict credit risk. The authors will concentrate on the latter in this paper. With application scoring, there is a basic choice whether to accept a prospect as a customer, or to decline him or her, based on some credit risk score. When the risk is too high, the applicant is rejected. As in most practical 
applications, many decision-making parameters are uncertain or even unknown, and most of the parameters vary over time. Such parameters include the cost of making a wrong decision (and the profit for making a right one), and assumptions about the underlying patterns of the data. Usually a solution to the problem is accompanied by a sensitivity analysis to changes in the underlying assumptions.

Customers that (intentionally) elude periodical payments of their mobile phone subscriptions are responsible for a large loss of income for mobile operators. Credit-scoring models can help to identify customers with a high credit risk. Based on historical data of defaulters, a credit-risk score can be devised for prospects (ie new applicants). People with a high score, or risk, are refused as new customers. Credit scoring can help to bring down the costs of incorrectly accepting defaulters by locating them and recognising them at the application stage. But sometimes lame ducks slip through the mazes of the net, and, sometimes, creditworthy customers may be erroneously refused. Too strict a risk management policy may result in the refusal of a lot of good customers ('goods'), whereas too loose a policy results in the acceptance of many 'bads'. Between the two extremes of declination of all prospects and acceptance of all prospects lies the optimal policy.

\section{MODELS FOR CREDIT SCORING}

Let us denote the declination rate of a policy in terms of the percentage of prospects that are declined. A strictness of zero means that none of the prospects are declined. A declination rate of 100 per cent means the declination of all prospects, including the creditworthy. A data model is created to predict the credit risk (or conversely the 'creditworthiness') of a prospect, with every new prospect receiving a score. Using a declination rate of 10 per cent, the top 10 per cent of prospects are declined. In order to construct such a model, two things are necessary: first, historical information on defaulters and secondly, discriminating features of prospects. Such features include age, residence, income etc. The model is a projection of the historical patterns on the prospect's features (ie the features of historical customers who did not pay their subscriptions). This projection results in a credit risk score. Intuitively, the score becomes higher the more a prospect's features coincide with the features of historical defaults. An interesting question is which features should be known in advance in order to create an accurate prediction of the credit risk of a customer.

In this paper, the authors will assure that a feasible set of features and one or more models are available. The problem of choosing the optimal credit risk policy now breaks down to a) the choice of the declination rate of the policy and b) the choice of the model (if several are available). At different declination rates, different models can be optimal. Therefore, the choice for the best policy and the best model are usually made simultaneously. This is also the case in this paper, although for simplicity, the authors will restrict themselves to only a single model for illustration.

\section{THE CLASSIFICATION MATRIX}

The classical quality assessment measure for classification models is the classification matrix, also known as the confusion matrix. In the two-class case, it sets out the actual class labels against the predicted class labels from the model. An example is shown in Figure 1.

In this credit-scoring problem, the 
Predicted

\begin{tabular}{|c|c|c|c|}
\hline & & Goods & Bads \\
\hline \multirow{2}{*}{ Actual } & Goods & 80 & 40 \\
\hline & Bads & 10 & 20 \\
\hline
\end{tabular}

Figure 1: Example of a classification matrix

Predicted

\begin{tabular}{|c|c|c|c|}
\hline & & Goods & Bads \\
\hline \multirow{2}{*}{ Actual } & Goods & TG & FB \\
\hline & Bads & FG & TB \\
\hline
\end{tabular}

Figure 2: Terminology of a classification matrix

class labels are conveniently called 'bads' and 'goods'. Here, the 'bads' are the defaulting customers and the 'goods' are the creditworthy customers. Figure 1 makes clear that there are 150 customers classified in total, of which 120 are goods and 30 are bads. These are the marginal row totals in the matrix. Of the 120 goods, 80 are correctly identified as creditworthy, whereas 40 are falsely predicted as defaulters. These numbers are called the true goods (TG) and the false bads (FB), respectively. Of the 30 bads, 20 are correctly classified as defaulters, and ten are falsely predicted as goods. These are the true bads (TB) and the false goods (FG), respectively. Figure 2 shows these terms.

As mentioned before, the models used for credit scoring produce a score, or sometimes a probability, that a customer is a defaulter. This probability lies on the unit interval between 0 and 1. Using this probability, applicants are declined (when labelled as 'bad'), or accepted (when labelled 'good'). A cut-off point is used to decide how many prospects are declined. For instance, a cut-off of 0.8 results in the declination of applicants with a credit risk probability of 0.8 or higher. The cut-off for this model is directly related to the declination rate of the policy. Higher cut-offs result in a smaller number of refusals, and thus in a smaller declination rate of the policy. Strictly speaking, the relationship between the cut-off and the declination rate is monotonic and surjective, which means that the declination rate may remain equal as the cut-off is changed.

Traditionally a cut-off of 0.5 is used, but in practical applications it is adjusted dynamically using the returns (in terms of revenue or profit) of the corresponding policy. This usually results in a much lower cut-off. A cut-off of 1 will result in the acceptance of all prospects. As the cut-off is gradually lowered, more and more customers are declined. So, the 'balance' in the classification matrix shifts from the first to the second column as the cut-off level decreases. Suppose the classification matrix in Figure 1 was the result of 
classifying the credit scores from a model using a cut-off of 0.3 . This means that the 60 customers predicted as 'bad' all have a score of 0.3 or higher, and the remaining 90 have a score below 0.3 Also, a cut-off level of 0.3 does not necessarily mean that 70 per cent of customers are classified as 'bad'.

The credit risk probabilities are not uniformly distributed on the unit interval, and in practice they are heavily concentrated around a low value with a strong skewness to the right. This means that there are a lot of prospects with a low probability (for instance 0.1 ), and only few with a higher probability. In this case, it is clear that 40 per cent (ie 60 out of the 150) of the prospects are labelled 'bad'. Therefore, the declination rate corresponding to the cut-off of 0.3 is 40 per cent.

\section{QUALITY ASSESSMENT}

Just how good is the policy using a declination rate of 40 per cent corresponding to Figure 1? Several quality measures have been proposed, ${ }^{2}$ with the classical ones being:

- Accuracy, computed as the percentage of correct predictions from the classification matrix;

$$
\text { accuracy }=\frac{T G+T B}{T G+F B+F G+T B}
$$

- Recall, computed as the fraction of true bads 'recalled' by the model;

$$
\text { recall }=\frac{T B}{F G+T B}
$$

- Precision, computed as the degree of 'purity' in the prediction of bads;

precision $=\frac{T B}{F B+T B}$
The accuracy measure has lost its importance in practical applications, because of its counterintuitive properties. ${ }^{3}$ Consider, for example, the accuracy of the classification matrix in Figure 1 and the accuracy of the (trivial) classification of every customer as negative (ie a declination rate of zero, with every applicant being labelled as 'good'). For Figure 1, the accuracy is $100 / 150$, whereas the trivial classification appears 'better' on the basis of its accuracy of $120 / 150$ (we correctly identify the 120 goods). This property of the accuracy measure occurs in the presence of 'imbalanced data'. In this case, imbalanced data means that the largest class (the goods) heavily outnumbers the smallest class (the bads). Here there are 30 bads versus 120 goods, and in many practical applications the bads-to-goods ratio is even lower. In recent years, extensive research on the problem of imbalanced data has appeared. ${ }^{4-11}$ The ROC to be discussed later in this paper, is one of the most promising results.

The problem with the accuracy classification might be circumvented at first sight by dynamically adjusting the cut-off level, until a maximum value for the accuracy is reached. Unfortunately, due to the imbalance problem, the trivial classification typically yields the maximum accuracy, so this approach is of little practical use. But the idea of plotting the quality measure against different values for the cut-off (or, equivalently, at different declination rates) has been adopted for the other two quality measures, recall and precision. The resulting graphs are called the captured response charts (CRCs), which are based on the recall measure, and the response charts, which are based on the precision measure. Two example charts are shown in Figures $3 a$ and $3 b$.

At a declination rate of 40 per cent, the values for recall and precision are 


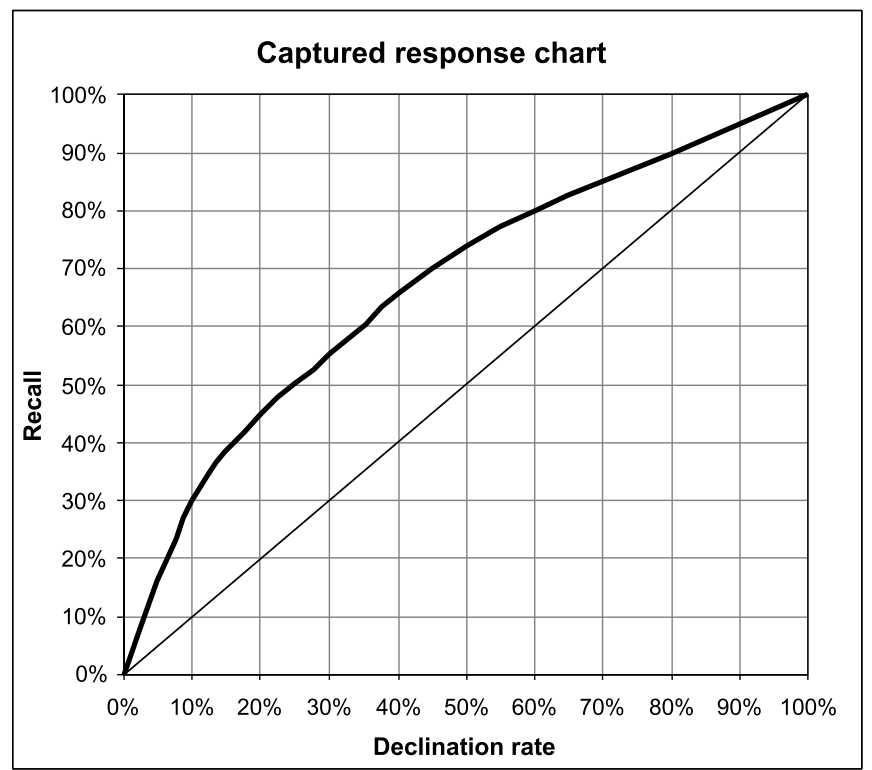

Figure 3a: Captured response chart

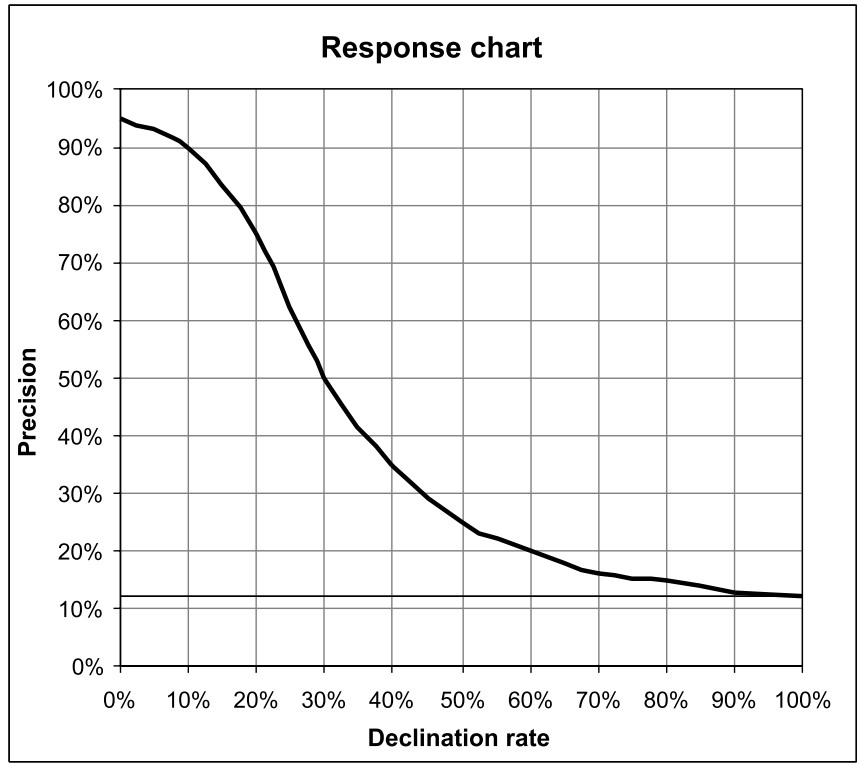

Figure 3b: Response chart

respectively 66 per cent and 33 per cent in Figures $3 \mathrm{a}$ and $3 \mathrm{~b}$. Both charts can be used to assess the quality of different models at different declination rates. Recall is only concerned with the number of false goods (ie incorrectly accepted defaulters), whereas precision is only concerned with the number of false bads (ie creditworthy customers that are incorrectly refused). It would be best for the quality measure to incorporate both types of model errors, since both are important. Note that accuracy does include both the false bads and the false 
Table 1: Cost and revenue calculations

\begin{tabular}{lll} 
& Cost & Revenue \\
\hline True goods & $\begin{array}{l}36 \begin{array}{c}\text { months } \times 100 \text { minutes } \times 80 \% \text { of } € 0.30 \\
=€ 864+€ 150=€ 1,014\end{array} \\
\text { False bads }\end{array}$ & $\begin{array}{c}36 \text { months } \times 100 \text { minutes } \times € 0.25 \\
=€ 1,080+€ 45=€ 1,125\end{array}$ \\
False goods & $\begin{array}{l}3 \text { months } \times 100 \text { minutes } \times 80 \% \text { of } € 0.30 \\
=€ 72+€ 150=€ 222\end{array}$ & nil \\
& nil & nil \\
\hline
\end{tabular}

goods, but treats these as equally objectionable (this is in fact the primary reason why the trivial classification results when accuracy is the quality measure of choice). It would also be good to use a quality measure that incorporates both the error of omission (ie incorrectly accepting a defaulter) and the error of commission (ie incorrectly refusing a nondefaulter), but at different weights. A common way to proceed is to incorporate costs or revenues to each of the classification matrix's cells.

\section{INCORPORATING COSTS}

To understand the use of costs and revenues, and as a running example of the later discussion on ROC analysis, consider a hypothetical mobile operator called MobPhone. A cost and revenue matrix can be constructed by making some assumptions.

A1 If applicants are accepted, they will pay a once-only hook up charge of $€ 45$.

A2 New applicants receive a purchase discount on a new handset with an average saving of $€ 150$.

A3 Customers are assumed to use 100 minutes per month, on average.

A4 The average per minute charge is $€ 0.30$
A5 Of this, 80 per cent is made up of MobPhone's costs (ie the net margin on a minute's call time is $€ 0.05$, on average).

A6 On average, customers will stay for three years (including contract and 'free-to-go' period).

A7 Defaulting customers will never pay any bills and will be disconnected after a period of three months after acceptance.

A8 Of all applicants, 20 per cent are defaulters.

The computations for cost and revenues are shown in Table 1. It is important to keep in mind that only actual costs and revenues are used.

Figure 4 shows the profit matrix resulting from the cost and revenue calculations. The goal is to maximise profit. The total profit for a policy is found by multiplying every cell in the classification matrix by its corresponding cell in the profit matrix. The total profit is given by

$$
\begin{aligned}
\text { Total profit }= & 111 \cdot T G+0 \cdot F B \\
& +(-177) \cdot F G+0 \cdot T B
\end{aligned}
$$

Having built a credit scoring model, the values for $T G, F B, F G$ and $T B$ are known for a given declination rate. Therefore, the objective is to maximise 
Predicted

\begin{tabular}{|c|c|c|c|}
\hline & & Goods & Bads \\
\hline \multirow{2}{*}{ Actual } & Goods & $€ 111$ & $€ 0$ \\
\hline & Bads & $€ 177$ & $€ 0$ \\
\hline
\end{tabular}

Figure 4: Profit matrix

the profit function across all declination rates for a model. But, when three is a choice between several models, the optimisation process should also choose the best model.

Letting $M$ denote the set of available models, and $S_{m}$ the set of possible values for the declination rate (between 0 and 1) for model $m \in M$ with corresponding values for $T G, F B, F G$ and $T B$. Then, for a certain model $m \in M$, the optimisation goal is

$$
\max _{m \in M, S_{m}} 111 . T G+(-177) \cdot F G
$$

The total profit resulting from the model corresponding to Figure 1 is

$$
\begin{aligned}
\text { Total profit } & =111 \cdot 80+(-177) \cdot 10 \\
& =7,110
\end{aligned}
$$

It would be quite a formidable task to evaluate all models at hand for every value of the declination rate in order to obtain the solution to the optimisation criterion. To make things even worse, it is very plausible that there is some level of uncertainty in the assumptions made. What will happen to the solution of the optimisation problem if the assumptions A1-A8 were only speculative. In practice, some simplifying generalisations or estimations about costs, revenues, detection times or contract periods will be made. In practice it is impossible to compare thousands of classification matrices for their sensitivity to changes in the underlying assumptions. ROC analysis provides a useful tool for this problem. ${ }^{12}$

\section{ROC ANALYSIS}

The ROC has also been used in signalling theory, where it is called the Receiver Operating Characteristic, and in medical applications. ${ }^{13,14}$ It was introduced in the artificial intelligence community in the 1980 s, but has attracted much attention in research on the imbalanced data problem over recent years. ${ }^{15}$ The ROC curve is comparable to that of the response chart and captured response chart in Figures $3 \mathrm{a}$ and $3 \mathrm{~b}$, in the sense that it reflects the quality of a model at different cut-offs, or rather, at different declination rates. One difference is that in both the response and the captured response charts the quality measures are plotted directly against their corresponding declination rate. In the ROC curve, one measure of quality is set out against another, both evaluated at the same declination rate. These measures are the true bads rate (TBR) and the false bads rate (FBR), computed as:

$$
\begin{aligned}
& T B R=T B /(T B+F G) \\
& F B R=F B /(F B+T G)
\end{aligned}
$$




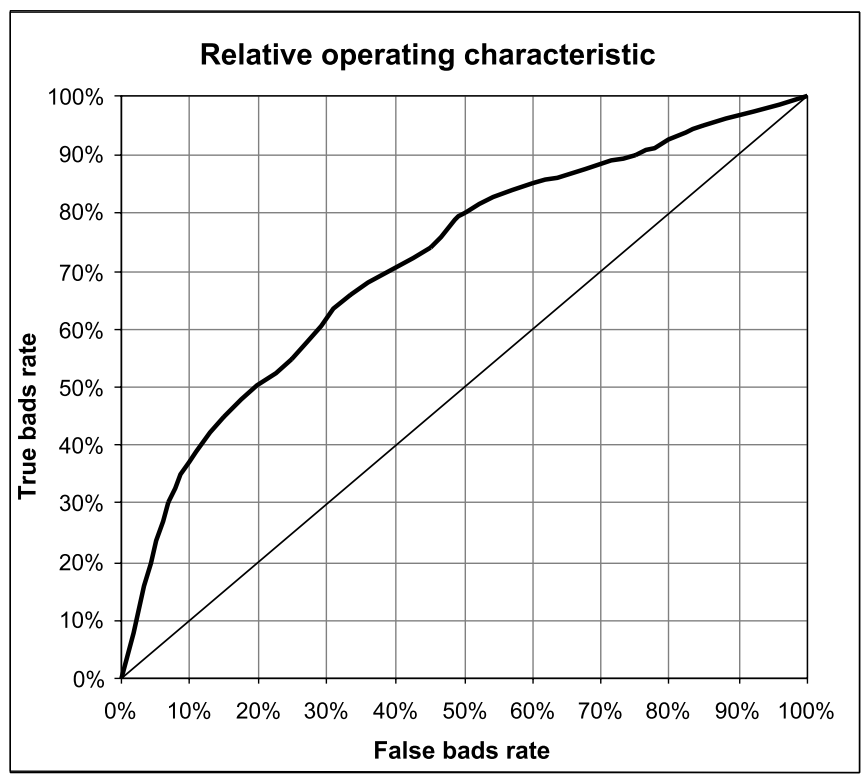

Figure 5: Relative operating characteristic

The TBR is actually equal to the recall quality measure given in the captured response chart. The vertical axes are the same for the captured response chart and the ROC chart. The horizontal axes differ. A sample ROC is shown in Figure 5.

It is clear that the curve looks like the one in the captured response chart, but it is slightly shifted upwards. Although not necessarily so, the ROC will usually lie above the captured response chart. The response and captured response charts both have the virtue that the declination rate can be read directly from the figure. The classification from Figure 1 corresponds to the point (33 per cent; 66 per cent) in the ROC chart, but there is no possibility of infering the 'strictness' level (40 per cent) from this point, without referring to the underlying classification matrix. It would require both the total number of actual bads and the total number of actual goods to compute the declination rate from a point on the ROC curve. Letting $G$ denote the number of actual goods $(T G+F B)$ and $B$ the actual number of bads $(F G+T B)$, then

'declination rate' $=$

$$
(T B R \cdot B+F B R \cdot G) /(B+G)
$$

Intuitively, within the setting of credit scoring, the TBR can be looked upon as the fraction of defaulters correctly declined at application, and the FBR as the fraction of non-defaulters incorrectly declined. If the declination rate of defaulters (TBR) is increased, it will usually result in higher declination of non-defaulters (FBR). Since both denominators in TBR and FBR are constant (they are equal to $B$ and $G$, respectively), increasing the declination rate so that one extra applicant is refused, means that either $T B$ or $F B$ increases (since the refused prospect is either a defaulter or not). This implies that the ROC curve is in fact a stepwise function, since it can only move vertically upwards when an extra defaulter is declined, or strictly horizontally rightwards when an extra non-defaulter is declined. This also implies that points further from the 
Predicted

\begin{tabular}{|c|c|c|c|}
\hline & & Goods & Bads \\
\hline \multirow{2}{*}{ Actual } & Goods & $p(T G)$ & $p(F B)$ \\
\hline & Bads & $p(F G)$ & $p(T B)$ \\
\hline
\end{tabular}

Figure 6: Profit matrix

origin correspond to stricter policies. Yet it may seem unclear why the ROC should be used when the user-friendlier response and captured response charts are at hand. Thus, with the profit matrix at hand, the ROC curve shows us an interesting way to tackle the profit maximisation problem.

\section{ISO-PROFIT LINES}

In order to obtain some useful properties of the ROC curve, it is necessary to make some computations. The authors' strategy is the following. Suppose there are two randomly chosen, but fixed, points in ROC space. Denote the points $\left(T B R_{1}, F B R_{1}\right)$ and $\left(T B R_{2}, F B R_{2}\right)$, with

$$
\begin{aligned}
& T B R_{k}=T B_{k} /\left(T B_{k}+F G_{k}\right)=T B_{k} / B \\
& F B R_{k}=F B_{k} /\left(F B_{k}+T G_{k}\right)=F B_{k} / G
\end{aligned}
$$

for $k=1,2$. We want to know the relationship between the two points when both points yield equal profits. Figure 6 shows the corresponding profit matrix.

Typically, $p(T G) \geq 0$ and $p(T B) \geq 0$ (since these are the profits associated with a correct classification), and $p(F B) \leq 0$ and $p(F G) \leq 0$ (since these are the costs, or negative profits, associated with an incorrect classification). The total profit for point $k$ is given by

$$
\begin{aligned}
& P_{k}=T G_{k} \cdot p(T G)+F B_{k} \cdot p(F B)+F G_{k} . \\
& p(F G)+T B_{k} \cdot p(T B)
\end{aligned}
$$

Or, expressed in terms of ROC coordinates,

$$
\begin{aligned}
& P_{k}=G \cdot\left(1-F B R_{k}\right) \cdot p(T G) \\
& +G \cdot F B R_{k} \cdot p(F B)+B \cdot\left(1-T B R_{k}\right) . \\
& p(F G)+B \cdot T B R_{k} \cdot p(T B) .
\end{aligned}
$$

Collecting terms results in

$$
\begin{aligned}
& P_{k}=G \cdot p(T G)+B \cdot p(F G)+G \cdot F B R_{k} \\
& {[p(F B)-p(T G)]+B \cdot T B R_{k} .} \\
& {[p(T B)-p(F G)] .}
\end{aligned}
$$

When equalling the formulae for $k=1$ and $k=2$, the first two terms cancel out, giving

$$
\begin{aligned}
& G \cdot F B R_{1} \cdot[p(F B)-p(T G)]+B \cdot T B R_{1} \\
& {[p(T B)-p(F G)]=G \cdot F B R_{2} .} \\
& {[p(F B)-p(T G)]+B \cdot T B R_{2} .} \\
& {[p(T B)-p(F G)] .}
\end{aligned}
$$

Shifting terms results in

$$
\begin{aligned}
& G \cdot[p(F B)-p(T G)] \cdot\left(F B R_{2}-F B R_{1}\right) \\
& =B \cdot[p(F G)-p(T B)] \cdot\left(T B R_{2}-T B R_{1}\right) .
\end{aligned}
$$

Rearranging terms finally gives

$$
\begin{aligned}
& \frac{T B R_{2}-T B R_{1}}{F B R_{2}-F B R_{1}} \\
& \quad=\frac{(1-\pi)}{\pi} \cdot \frac{p(F B)-p(T G)}{p(F G)-p(T B)}
\end{aligned}
$$

where $\pi=B /(B+G)$. In other words, given the profit matrix in Figure 6, and 


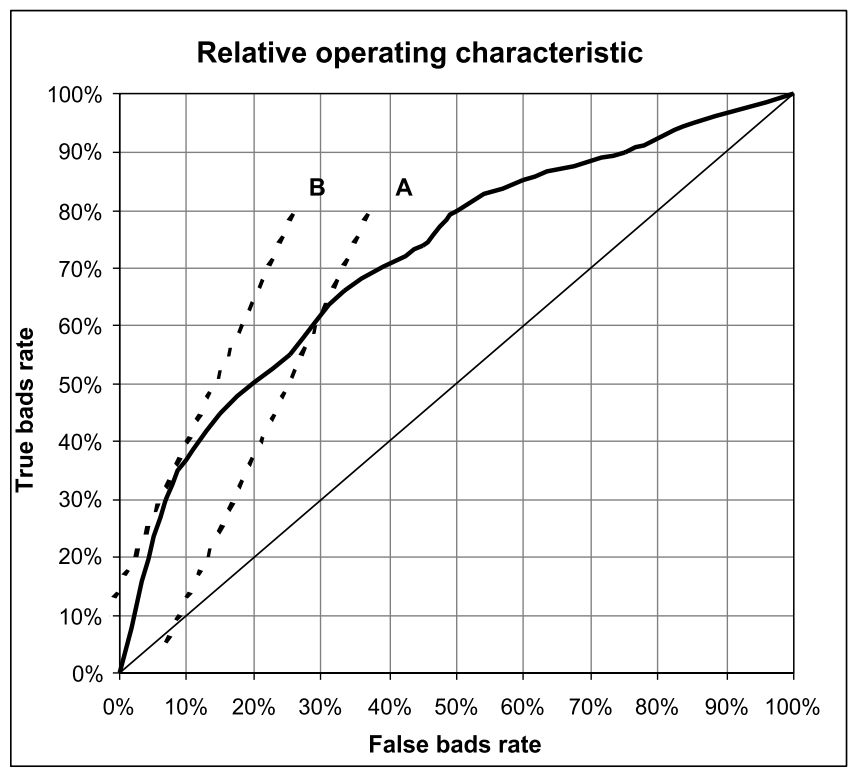

Figure 7: ROC plus iso-profit lines

the proportion of bads in the population $\pi$, all points having equal profits lie on a straight line with slope given by the right hand side of (2). These lines are called the iso-profit lines. Using the profits in Figure 4 and remembering that the fraction of fraudulent prospects was $\pi=20$ per cent (assumption A8), then for this example, the following slope is obtained:

$$
\text { slope }=\frac{(1-0.20)}{0.20} \cdot \frac{(0-111)}{((-177)-0)} \approx 2.5
$$

The iso-profit line can be placed anywhere in the ROC chart, and it can be shifted in any direction. Points to the left of the line will have a higher profit than points on or below the line. This is seen by taking a point $\left(T B R_{1}, F B R_{1}\right)$ on the iso-profit line and a point $\left(T B R_{2}, F B R_{2}\right)$ above the line. Assume that $F B R_{1}<F B R_{2}$ and $T B R_{1}=T B R_{2}$. From (1) we see that the profit increases in the first point if $p(T G)>p(F B) \quad$ (ie the profit for accepting a non-defaulter is higher than the profit for declining a non-defaulter), which will almost always be so in practice. For two iso-profit lines, this means that the one lying left of the other has the highest total profit.

Figure 7 shows two iso-profit lines with a slope of 2.5. Since line B lies left of line $\mathrm{A}$, it represents points yielding a higher total profit. This means that the point ( 8 per cent, 35 per cent), the intersection of iso-profit line $\mathrm{B}$ and the ROC curve, has a higher profit than the point (30 per cent, 60 per cent), at the intersection of iso-profit line $\mathrm{A}$ and the ROC curve. Since the iso-profit line cannot be shifted any further left and still intersect the ROC curve, the point ( 8 per cent, 35 per cent) is optimal. This means that at this point, the total profit is maximal under the assumptions made. Translated into the declination rate, a TBR of 35 per cent means the correct decline of 35 per cent of the 40 defaulters (14 applicants) and the incorrect decline of 8 per cent of the 120 actual goods (about 7 customers). So, 21 of 150 applicants are declined, which means a declination rate of approximately 14 per cent. 
Table 2: Cost and revenue calculations

\begin{tabular}{llc}
\hline Cost & Revenue \\
\hline True goods & $\begin{array}{c}36 \begin{array}{c}\text { months } \times 75 \text { minutes } \times 80 \% \text { of } € 0.30 \\
=€ 648+€ 150=€ 798\end{array} \\
36 \begin{array}{c}\text { months } \times 150 \text { minutes } \times 80 \% \text { of } € 0.30 \\
=€ 1,296+€ 150=€ 1,446\end{array}\end{array}$ & $\begin{array}{c}36 \text { months } \times 75 \text { minutes } \times € 0.30 \\
=€ 810+€ 45=€ 855\end{array}$ \\
& $\begin{array}{c}\text { months } \times 150 \text { minutes } \times € 0.30 \\
=€ 1,620+€ 45=€ 1,665\end{array}$ \\
\hline
\end{tabular}

\section{SENSITIVITY TO CHANGES IN THE ASSUMPTIONS}

As mentioned before, the exact parameters determining the values in the profit matrix are often estimated or generalised. When using the classification matrix, the response chart or the captured response chart, the impact of a change in profits on the solution of the problem can only be assessed by recomputing the new solution and corresponding profit. This will be quite a formidable task, if sensitivity analysis has to be performed. In ROC analysis, this is made much easier. Consider the example in which assumption A3 (the average number of minutes called by non-defaulters) is altered. If the average call duration is uncertain and the average of 100 minutes per month can be replaced by the range $[75,150]$, the cost and revenue for true negatives can be calculated as shown in Table 2 .

This means that $57 \leq p(T G) \leq 219$. The rest of the profit matrix remains unchanged. As a result, from formula (2) it can be seen that the slope ranges from 1.4 for $p(T G)=57$ to 4.1 for $p(T G)=219$. Figure 8 shows what happens as the iso-profit line tilts from a slope of 4.1 to a slope of 1.4. For the 150 minutes case (ie the slope of 4.1), the point ( 5 per cent, 25 per cent) is optimal. For the 75 minutes case, the optimum lies at (15 per cent, 45 per cent). The corresponding declination rates are 9 per cent and 21 per cent, respectively. So the authors' policy declines fewer applicants when the average call duration increases. This makes sense, since higher call duration increases the profitability of customers, meaning that it will be better to accept more applicants and just accept the number of defaulters. The increase in the profitability of creditworthy customers makes up for the lack of effectiveness in credit risk management.

In the example given in Figure 8, ROC analysis showed that as the average call duration varies between 75 and 150 minutes, the optimal policy would lie between 9 per cent and 21 per cent. The same analysis can be made subject to changes to other assumptions. Table 3 shows the effect on the slope of the iso-profit line when one of the assumptions $\mathrm{A} 1-\mathrm{A} 8$ changes (ceteris paribus). Remember that steeper iso-profit lines favour policies with a lower declination rate, and lower iso-profit lines favour policies with a higher declination rate.

\section{COMPARISON OF THE ROC TO OTHER MEASURES}

The close relationship between the ROC and the CRC (Figure 3a) has already been mentioned. Whereas the CRC shows classification accuracy for the 'bads', it does not show the imprecision on the 'goods'. ROC shows both explicitly. Furthermore - and this is the biggest advantage of ROC analysis in the authors' opinion - the iso-profit curves are straight lines in ROC space. For $\mathrm{CRC}$ and other measures, the iso-profit 


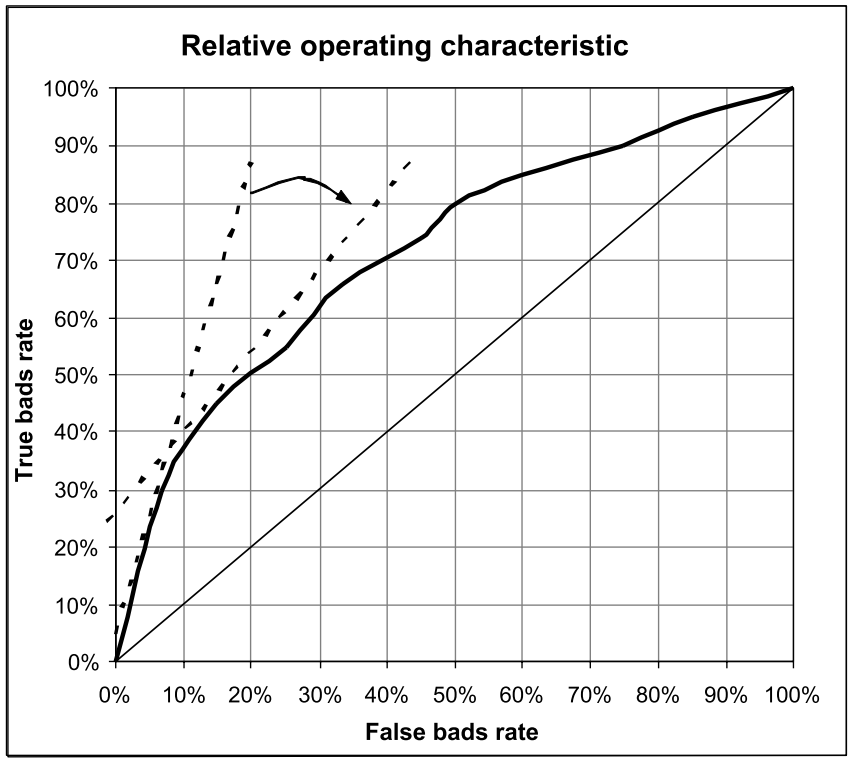

Figure 8: Sensitivity analysis in ROC analysis

Table 3: Sensitivity analysis

\begin{tabular}{|c|c|c|}
\hline & Change & Effect \\
\hline A1 & Increasing (decreasing) hook up charges & $\begin{array}{l}\text { Increasing (decreasing) iso-profit line } \\
\text { lower (higher) declination rate }\end{array}$ \\
\hline $\mathrm{A} 2$ & lincreasing (decreasing) cost handset & $\begin{array}{l}\text { Decreasing (increasing) iso-profit line } \\
\text { higher (lower) declination rate }\end{array}$ \\
\hline A3 & Increasing (decreasing) average call duration & $\begin{array}{l}\text { Increasing (decreasing) iso-profit line } \\
\text { lower (higher) declination rate }\end{array}$ \\
\hline A4 & Increasing (decreasing) minute charge & $\begin{array}{l}\text { Decreasing (increasing) iso-profit line } \\
\text { higher (lower) declination rate }\end{array}$ \\
\hline A5 & Increasing (decreasing) cost per minute & $\begin{array}{l}\text { Decreasing (increasing) iso-profit line } \\
\text { higher (lower) declination rate }\end{array}$ \\
\hline A6 & Increasing (decreasing) average subscription period & $\begin{array}{l}\text { Increasing (decreasing) iso-profit line } \\
\text { lower (higher) declination rate }\end{array}$ \\
\hline A7 & Increasing (decreasing) time before suspension & $\begin{array}{l}\text { Decreasing (increasing) iso-profit line } \\
\text { higher (lower) declination rate }\end{array}$ \\
\hline A8 & Increasing (decreasing) percentage defaulters & $\begin{array}{l}\text { Decreasing (increasing) iso-profit line } \\
\text { higher (lower) declination rate }\end{array}$ \\
\hline
\end{tabular}

curves would not be straight lines, but actual curves. Consequently, changing the assumptions even slightly will change the iso-profit curves in unpredictable ways, making sensitivity analysis hazy.

\section{ASSUMPTIONS AND THE STRAIGHT ISO-PROFIT LINE PROPERTY}

In our calculations of the profit matrix (Figure 4), the same values for average monthly volume (assumption A3) were used for both the 'goods' and the 'bads' classes. In practice, it may well be that the features of bads and goods differ. For instance, a fraudulent customer might call more on average since he or she has no incentive to keep the phone bill to a low amount. This can be easily implemented in the analysis described above by using different averages for goods and bads in the corresponding cells of the profit matrix. A more complicated situation arises when it is believed that 


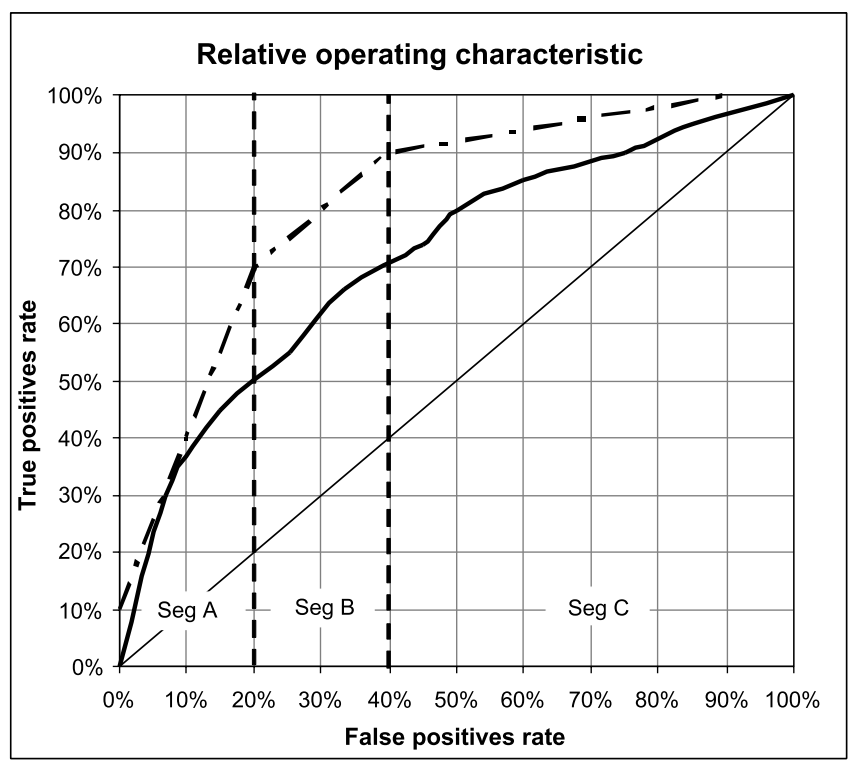

Figure 9: Segmented ROC analysis using a piecewise-linear iso-profit curve

the call volume (or any other value in the assumptions) varies with the risk score from our model (ie when monthly volumes are higher, the fraction of fraudulent customers increases). This would mean that the profits (and therefore also the slopes of the iso-profit lines) change at different points in ROC space. The iso-profit curve would then not be a straight line anymore, and the advantage of ROC analysis easily assessing sensitivity to the assumptions would disappear. The authors' suggestion is to first verify how strongly the values in the assumptions correlate with the model risk score. If the correlation proves reasonably low, ROC analysis as described previously can be used. If there is significant correlation, an option may be to segment the customer base based on risk score bands, and assume equal values within each segment, for instance, the segment of risk scores between 0.4 and 0.6 use 100 minutes, and the segment having band 0.2 to 0.4 use 80 minutes, and so on. The resulting iso-profit curve would be piecewise-linear (ie the iso-profit curve is a straight line within each segment), as can be seen in Figure 9.

\section{CONCLUSION}

The authors have described the use of ROC analysis for decision-making under uncertain circumstances. By comparison with other, more traditional, quality measures, the ROC is capable of showing both the optimal point and the sensitivity to changes in the underlying cost and distribution assumptions. This paper was written from the perspective of a mobile telephone operator using credit scoring to detect potential defaulters. The assumptions made mainly concerned cost and profit premises. This is only one of many applications in which ROC analysis can be used. ROC analysis is appealing because of its capability to perform sensitivity analysis and decision policy optimisation in one figure. Although its compilation is less intuitive than that of some other measures, the advantages will compensate for this generously. 


\section{References}

1 Bolton, R. J. and Hand, D. J. (2001) 'Peer group analysis - Local anomaly detection in longitudinal data', Technical report, Department of Mathematics, Imperial College, London, UK.

2 Kubat, M. and Matwin, S. (1997) 'Addressing the curse of imbalanced data sets: one-sided sampling', Proceedings of the International Conference On Machine Learning, Nashville, Tennessee, 12th July, 1997, Morgan Kaufmann, pp. 179-186.

3 Provost, F., Fawcett, T. and Kohavi, R. (1998) 'The case against accuracy estimation for comparing induction algorithms', Proceedings of the International Conference On Machine Learning, Madison, Wisconsin, 24th July, 1998, Morgan Kaufmann, pp. 445-453.

4 Kubat and Matwin (1997) op. cit.

5 Provost, Fawcett and Kohavi (1998) op. cit.

6 Japkowicz, N. (2000) 'Learning from imbalanced data sets: A comparison of various strategies', Proceedings of the AAAI'00 workshop on learning from imbalanced data sets, 30th July 2000, Austin, Texas, American Association for Artificial Intelligence, technical report WS-00-05, Menlo Park, CA.

7 Japkowicz, N. (2000) 'The class imbalance problem: Significance and strategies', Proceedings of the 2000 International Conference on Artificial Intelligence, Las Vegas, Nevada, 26th June, 2000, pp. 111-117.

8 Japkowicz, N. (2000) 'Concept learning in the presence of between-class and within-class imbalances', Proceedings of the 14th Conference of the Canadian Society for Computational Studies of Intelligence, 7th June, 2001, Ottawa, Canada, Springer, Germany, pp. 67-77

9 Pednault, E. P. D., Rosen, B. K. and Apte, C. (2000) 'Handling imbalanced data sets in insurance risk modeling', IBM Research, TR-RC-21731.

10 Laurikkala, J. (2001) 'Improving identification of difficult small classes by balancing class distribution', Technical Report April 2001(2), Department of Computer and Information Sciences, University of Tampere.

11 Weiss, G. M. and Provost, F. (2001) 'The effect of class distribution on classifier learning', Technical Report, ML-TR-43, Rutgers University, New Jersey.

12 Hand, D. J. and Henley, W. E. (1997) 'Statistical classification methods in consumer credit scoring: a review', Journal of the Royal Statistical Society, Series A, Vol. 160, pp. 523-541.

13 Egan, J. P. (1975) 'Signal detection theory and ROC analysis', Series in cognition and perception, Academic Press, New York.

14 Swets, J. A. (1988) 'Measuring the accuracy of diagnostic systems', Science, Vol. 240, pp. 1285-1293.

15 Provost, F. and Fawcett, T. (2001) 'Robust classification for imprecise environments', Machine Learning, Vol. 42, pp. 203-231. 